\title{
SELF-SIMILAR STATIC SOLUTIONS ADMITTING A TWO-SPACE OF CONSTANT CURVATURE
}

\author{
J. Carot, and A.M. Sintes \\ Departament de Física \\ Universitat de les Illes Balears \\ E-07071 Palma de Mallorca. SPAIN
}

\begin{abstract}
A recent result by Haggag and Hajj-Boutros [1] is reviewed within the framework of self-similar space-times, extending, in some sense, their results and presenting a family of metrics consisting of all the static spherically symmetric perfect fluid solutions admitting a homothety.
\end{abstract}

PACS numbers: 04.20Jb, 02.40+m, 98.80Dr 
In a recent paper, Haggag and Hajj-Boutros [1] presented a static, spherically symmetric perfect fluid solution with a stiff-matter type equation of state (i.e.: $p=\mu$ ). By means of a few clever changes of coordinates, the authors reduce the problem to that of solving a non-linear, second order differential equation, whose polynomic solutions they investigate showing that only three such solutions exist, two of them being vacuum (flat Minkowski space-time and Schwarzschild solution) and the third one being that leading to the new metric referred to above, henceforth called HHB solution.

The purpose of this letter is to give all the static, spherically symmetric perfect fluid solutions admitting a homothety. This family can be completely characterized by means of a real parameter $\gamma$ (arising quite naturally from the equation of state for these fluids, see below), which must be in the interval $[1,2]$ in order to satisfy energy conditions. The two limiting values of $\gamma$, namely $\gamma=1$ and $\gamma=2$ correspond to Minkowski flat space-time and to the HHB solution respectively.

A few remarks concerning the similarity group and its action are in order here. It is a well known fact that an $r$-parameter group of homotheties $H_{r}$ (in which at least one proper homothety exists) always admits an $(r-1)$-parameter subgroup of isometries $G_{r-1}$. Now, the maximal dimension of the group of homotheties that a perfect fluid space-time may admit is $r=7$, in which case it is one of the special RobertsonWalker space-times [2], and therefore they are all known. The case $r=6$ is not compatible with an energy-momentum tensor of the perfect fluid type; thus, apart from the special Robertson-Walker solutions mentioned above, the highest dimension of the group of homotheties that a perfect fluid space-time may admit is $r=5$. In such case, the associated isometry subgroup $G_{4}$ has necessarily 3-dimensional nonnull orbits [2]. Notice that this is precisely the case we are interested in. We shall not treat here the case in full generality, namely; studying all perfect fluid space-times 
admitting an $H_{5}$ of homotheties, since this would be beyond the purpose of this letter, but we shall restrict ourselves to the case when the subgroup $G_{4}$ has timelike orbits $T_{3}$ and the subgroup $G_{3}$ that it necessarily contains [3] has two-dimensional orbits. Everything else follows from these assumptions and the field equations. For further information on groups of homotheties and related issues, we refer the reader to [4, 5] and [6].

We start with a space-time that contains a non-null two-space of constant curvature (i.e.: there exists a three-parameter isometry group $G_{3}$ acting on this two-space). In this case the orbits $V_{2}$ admit orthogonal surfaces in $M$ []]. By performing a coordinate transformation in the two-spaces orthogonal to the Killing orbits the space-time metric can be put into diagonal form:

$$
\begin{gathered}
d s^{2}=A^{2}(r, t)\left(-d t^{2}+d r^{2}\right)+B^{2}(r, t)\left(d \theta^{2}+f^{2}(\theta, k) d \phi^{2}\right) \\
f(\theta, k)= \begin{cases}\sin \theta & k=+1 \\
\theta & k=0 \\
\sinh \theta & k=-1 .\end{cases}
\end{gathered}
$$

where we have restricted ourselves to the case of spacelike Killing orbits, since perfect fluid and dust solutions cannot admit a group $G_{3}$ on two-dimensional timelike orbits [3].

Using the Jacobi identities and the fact that the Lie bracket of a proper homothetic vector field (HVF) and a Killing vector $(\mathrm{KV})$ is a $\mathrm{KV}$ it can be easily shown that the HVF $X$ must be of either one of the following forms:

$$
\begin{aligned}
(I) \quad X & =X^{t}(r, t) \partial_{t}+X^{r}(r, t) \partial_{r}, \quad k=-1,0,1 \\
(I I) \quad X & =X^{t}(r, t) \partial_{t}+X^{r}(r, t) \partial_{r}-\theta \partial_{\theta}, \quad k=0 .
\end{aligned}
$$

Now by using isotropic coordinates, one finds that static metrics can be expressed as 
[3]:

$$
d s^{2}=-A^{2}(r) d t^{2}+B^{2}(r)\left[d r^{2}+r^{2}\left(d \theta^{2}+f^{2}(\theta, k) d \phi^{2}\right)\right]
$$

where $\partial_{t}$ is the hypersurface orthogonal timelike KV.

In this coordinate chart, the HVF in (3) and (4) takes the following forms:

$$
\begin{aligned}
(I) \quad X & =n t \partial_{t}+R(r) \partial_{r}, \quad k=-1,0,1 \\
(I I) \quad X & =n t \partial_{t}+R(r) \partial_{r}-\theta \partial_{\theta}, \quad k=0,
\end{aligned}
$$

where $n$ is a constant.

The homothetic equation $\mathcal{L}_{X} g_{a b}=2 g_{a b}$ specified to the components $\mathrm{rr}$ and $\theta \theta$ of the metric (5), gives:

$$
R_{, r}-\frac{R}{r}-X_{, \theta}^{\theta}=0
$$

and integrating, one gets:

$$
\begin{aligned}
(I) \quad X & =n t \partial_{t}+q r \partial_{r}, \quad k=-1,0,1 \\
(I I) \quad X & =n t \partial_{t}+(-r \ln r+c r) \partial_{r}-\theta \partial_{\theta}, \quad k=0
\end{aligned}
$$

where $c$ and $q(\neq 0)$ are constants.

\section{Case (I)}

By means of the coordinate transformation $\hat{r}=r^{1 / q}$, the HVF and the metric can be written as

$$
\begin{gathered}
X=n t \partial_{t}+\hat{r} \partial_{\hat{r}} \\
d s^{2}=-\hat{A}^{2}(\hat{r}) d t^{2}+\hat{B}^{2}(\hat{r})\left[q^{2} d \hat{r}^{2}+\hat{r}^{2}\left(d \theta^{2}+f^{2}(\theta, k) d \phi^{2}\right)\right]
\end{gathered}
$$

The metric functions can be determined via the homothetic equations, that gives:

$$
\hat{B}=\text { constant }, \quad \hat{A} \propto \hat{r}^{1-n}
$$


Defining a new radial coordinate $r$ as $r=\hat{r} \hat{B}$, one can come to the following simple forms for $X$ and the metric:

$$
\begin{gathered}
X=n t \partial_{t}+r \partial_{r}, \\
d s^{2}=-r^{2(1-n)} d t^{2}+q^{2} d r^{2}+r^{2}\left(d \theta^{2}+f^{2}(\theta, k) d \phi^{2}\right) .
\end{gathered}
$$

\section{Case $(I I)$}

Imposition of the homothetic equations specified to the metric (5) and to the HVF (10), leads directly to:

$$
d s^{2}=-(-\ln r+c)^{2(n-1)} d t^{2}+\frac{b^{2}}{r^{2}(-\ln r+c)^{4}}\left[d r^{2}+r^{2}\left(d \theta^{2}+\theta^{2} d \phi^{2}\right)\right],
$$

$b$ and $c$ being constants.

From the expressions (15) and (16) of the metric, it is immediate to see that the components $t t$ of their respective Einstein tensors are negative for $k=-1$ and 0 (i.e.: hyperbolic and flat two-spaces) and therefore cannot verify energy conditions. Thus, it only remains to study the spherically symmetric case.

In this latter case, the field equations for a perfect fluid matter content lead to the metric:

$$
d s^{2}=-r^{4-4 / \gamma} d t^{2}+\left(\frac{\gamma^{2}+4 \gamma-4}{\gamma^{2}}\right) d r^{2}+r^{2}\left(d \theta^{2}+\sin ^{2} \theta d \phi^{2}\right),
$$

These metrics were already found, following a completely different approach, by Ibañez et al. [9], and particular cases of them can be also found in Misner et al. [10] (which are particular cases of Tolman class VI solutions). Some particular cases (when the HVF is orthogonal to the fluid flow) were also studied by Herrera et al.[11]. 
The matter variables being

$$
\begin{gathered}
\mu=\frac{1}{r^{2}}\left(\frac{4 \gamma-4}{\gamma^{2}+4 \gamma-4}\right), \\
p=(\gamma-1) \mu
\end{gathered}
$$

as one would have expected from $p$ and $\mu$ being functions of $r$ alone (and therefore, by the implicit function theorem, the fluid has a barotropic equation of state) and the space-time being self-similar [8]. The HVF takes then the form:

$$
X=\frac{2-\gamma}{\gamma} t \partial_{t}+r \partial_{r}
$$

These are all the static, spherically-symmetric self-similar perfect fluid solutions. They are shear-free and have null volume expansion since the four-velocity $u$ of the fluid is parallel to the timelike $\mathrm{KV}$, the vorticity is also zero (since $u$ is orthogonal to the orbits $S_{2}$ of the $G_{3}$ they contain); and the fluid has non-geodesic flow.

The particular case, $\gamma=2$, is the HHG solution $\| 1$ and in this case the HVF $X$ becomes orthogonal to the fluid four-velocity, and for $\gamma=1$ the space-time is obviously flat.

It is interesting to notice that static and self-similar solutions admitting a twospace of constant curvature can only be spherically symmetric (irrespectively of the matter content) and that an $H_{5}$ static space-time with a $G_{3}$ acting on spacelike orbits, necessarily contains an $H_{4}$. 


\section{References}

[1] S. Haggag and J. Hajj-Boutros, Class. Quantum. Grav., 11(1994)L69.

[2] G.S. Hall and J.D. Steele, Gen. Rel. Grav., 22(1990)457.

[3] D. Kramer, H. Stephani, M.A.H. MacCallum and E. Herlt. Exact Solutions of Einstein's Field Equations. Deutscher Verlag der Wissenschaften, Berlin (1980).

[4] G.S. Hall, Class. Quantum. Grav., 5(1988)L77.

[5] G.S. Hall, Gen. Rel. Grav., 20(1988)671.

[6] G.S. Hall, J. Math. Phys. 31(1990)1198.

[7] B.G. Schmidt, Z. Naturforsch. 22a(1967)1351.

[8] J. Wainwright, Self-similar solutions of Einstein's equations in Galaxies, Axisymmetric systems and Relativity, ed. M.A.H. MacCallum, Cambridge (1985) C.U.P.

[9] J. Ibañez and J.L. Sanz, J. Maths. Phys., 23(1982)1364.

[10] C.W. Misner and H.S. Zapolsky, Phys. Rev. Lett., 12(1964)635.

[11] L. Herrera, J. Jimenez, L. Leal, J. Ponce de Leon, M. Esculpi and V. Galina, J. Maths. Phys., 25(1984)3277. 